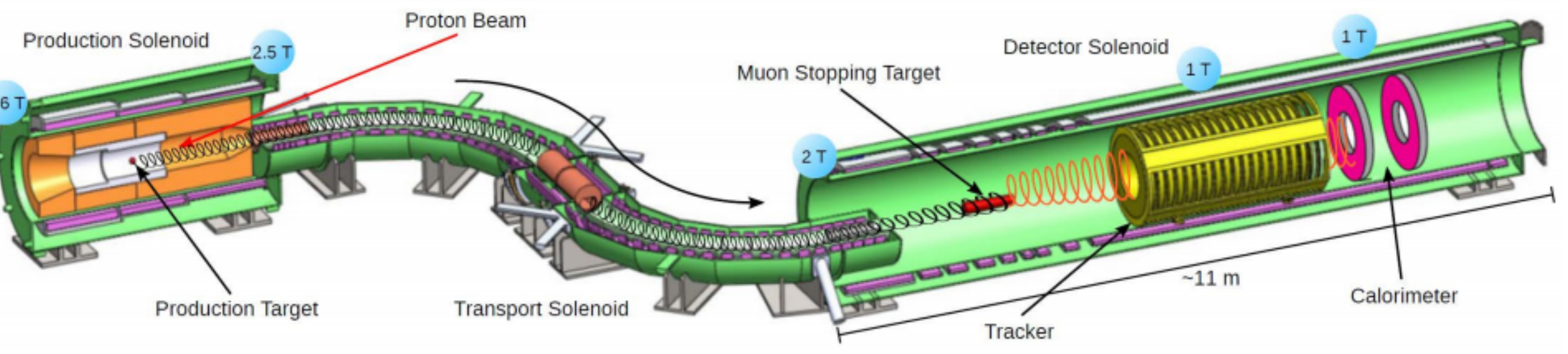

\title{
The Mu2e Experiment: \\ A Search for Charged Lepton \\ Flavor Violation
}

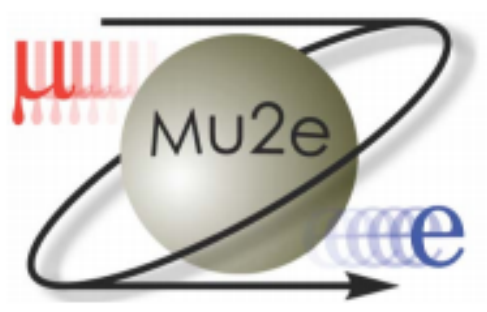

Dan Ambrose

University of Minnesota

Lake Louise Winter Institute 2019

This document was prepared by using the resources of the Fermi National Accelerator Laboratory (Fermilab), a U.S.

Department of Energy, Office of Science, HEP User Facility. Fermilab is managed by Fermi Research Alliance, LLC (FRA),

acting under Contract No. DE-AC02-07CH11359.

Feb 16, 2019 


\section{Charged Lepton Flavor Violation (CLFV)}

With neutrino mass, we know that lepton flavor is not conserved. The SM CLFV process would be :

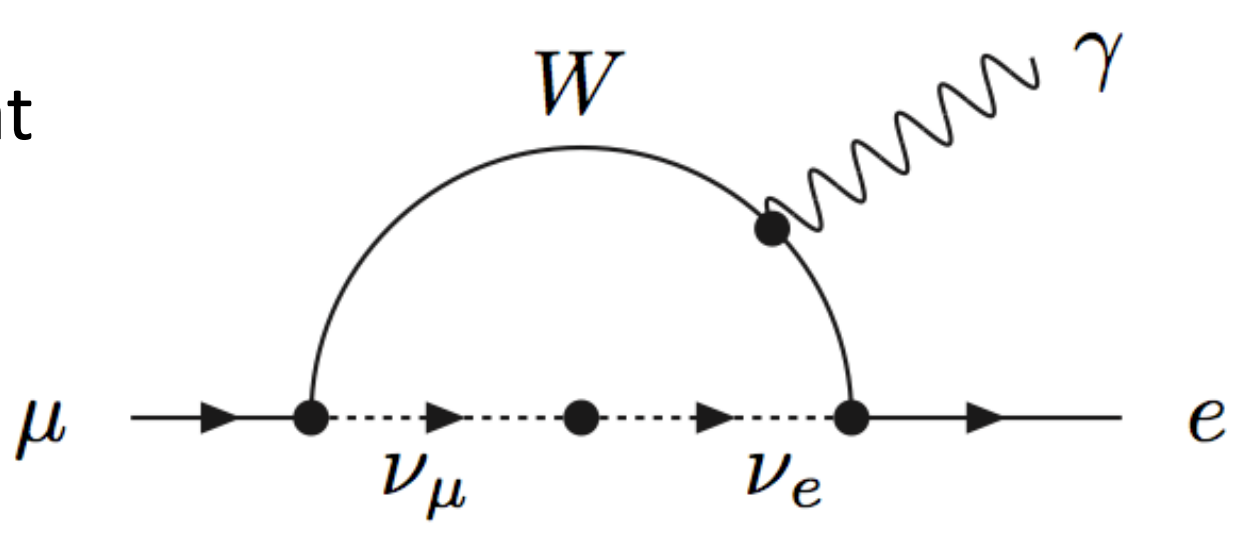

$$
\mathcal{B}(\mu \rightarrow e \gamma)=\frac{3 \alpha}{32 \pi}\left|\sum_{i=2,3} U_{\mu i}^{*} U_{e i} \frac{\Delta m_{1 i}^{2}}{M_{W}^{2}}\right|^{2}<10^{-54}{ }_{\text {* Physically unobservable }}
$$

Any observation of CLFV must be new physics! 


\section{Searching for CLFV}
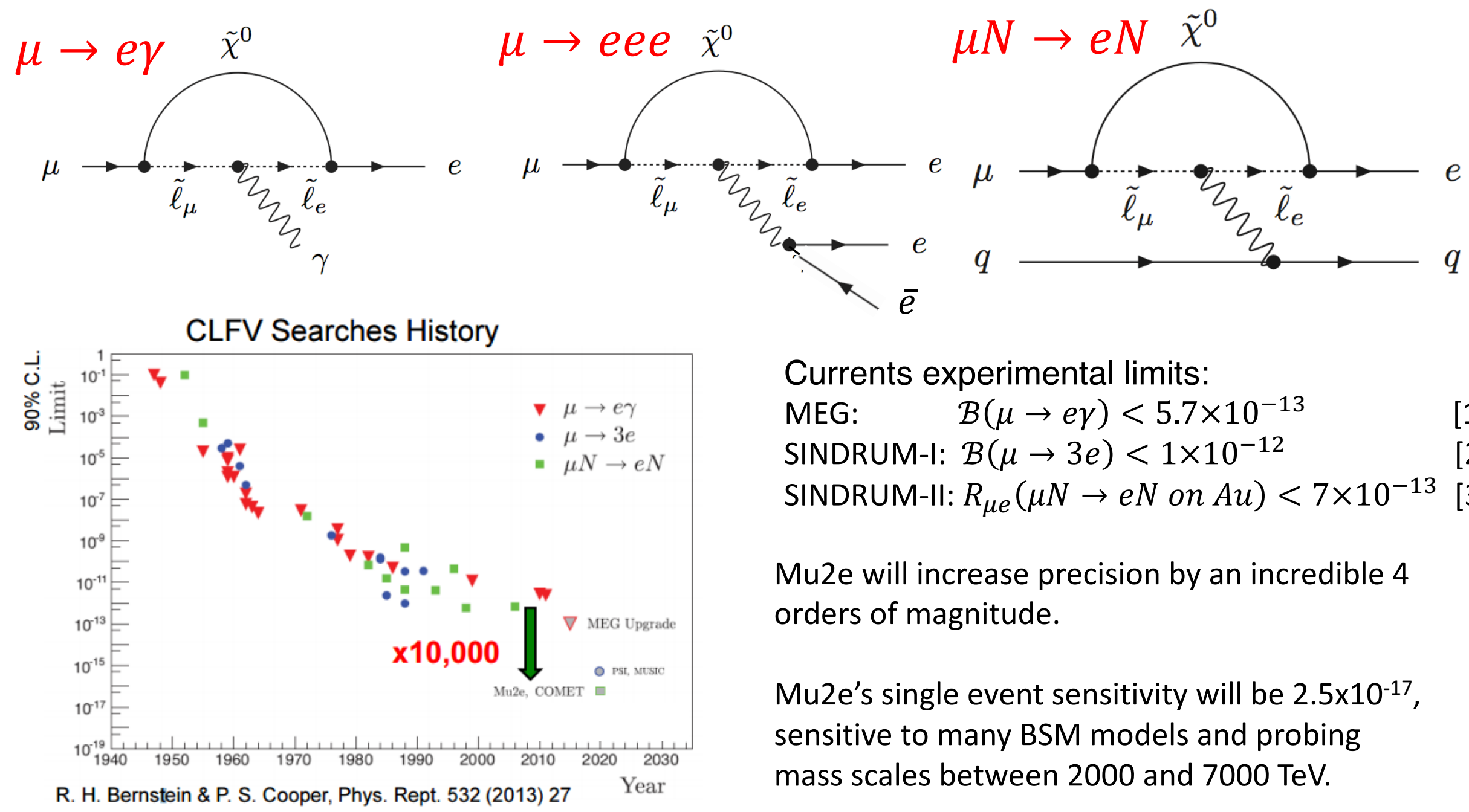

Currents experimental limits:

MEG: $\quad \mathcal{B}(\mu \rightarrow e \gamma)<5.7 \times 10^{-13}$ SINDRUM-I: $\mathcal{B}(\mu \rightarrow 3 e)<1 \times 10^{-12}$ SINDRUM-II: $R_{\mu e}(\mu N \rightarrow e N$ on $A u)<7 \times 10^{-13}$

Mu2e will increase precision by an incredible 4 orders of magnitude.

Mu2e's single event sensitivity will be $2.5 \times 10^{-17}$, sensitive to many BSM models and probing mass scales between 2000 and $7000 \mathrm{TeV}$. 


\section{Mu2e's Concept}

- Use Fermilab's proton beam to create a beam of low momentum muons

- Stop the muons in an Aluminum target ( $10^{18}$ over 3 yrs)

- Detectors which look for mono-energetic electrons consistent with $\mu \mathrm{N} \rightarrow \mathrm{eN}$ and differentiate background

- We measure :

$$
R_{\mu e}=\frac{\mu^{-}+N(A, Z) \rightarrow e^{-}+N(A, Z)}{\mu^{-}+N(A, Z) \rightarrow \nu_{\mu}+N(A, Z-1)}
$$

Numerator:

Muon to electron conversion in the presence of a nucleus Denominator:

Nuclear captures of muonic Al atoms 


\section{Creating and Decaying Muonic Atom}

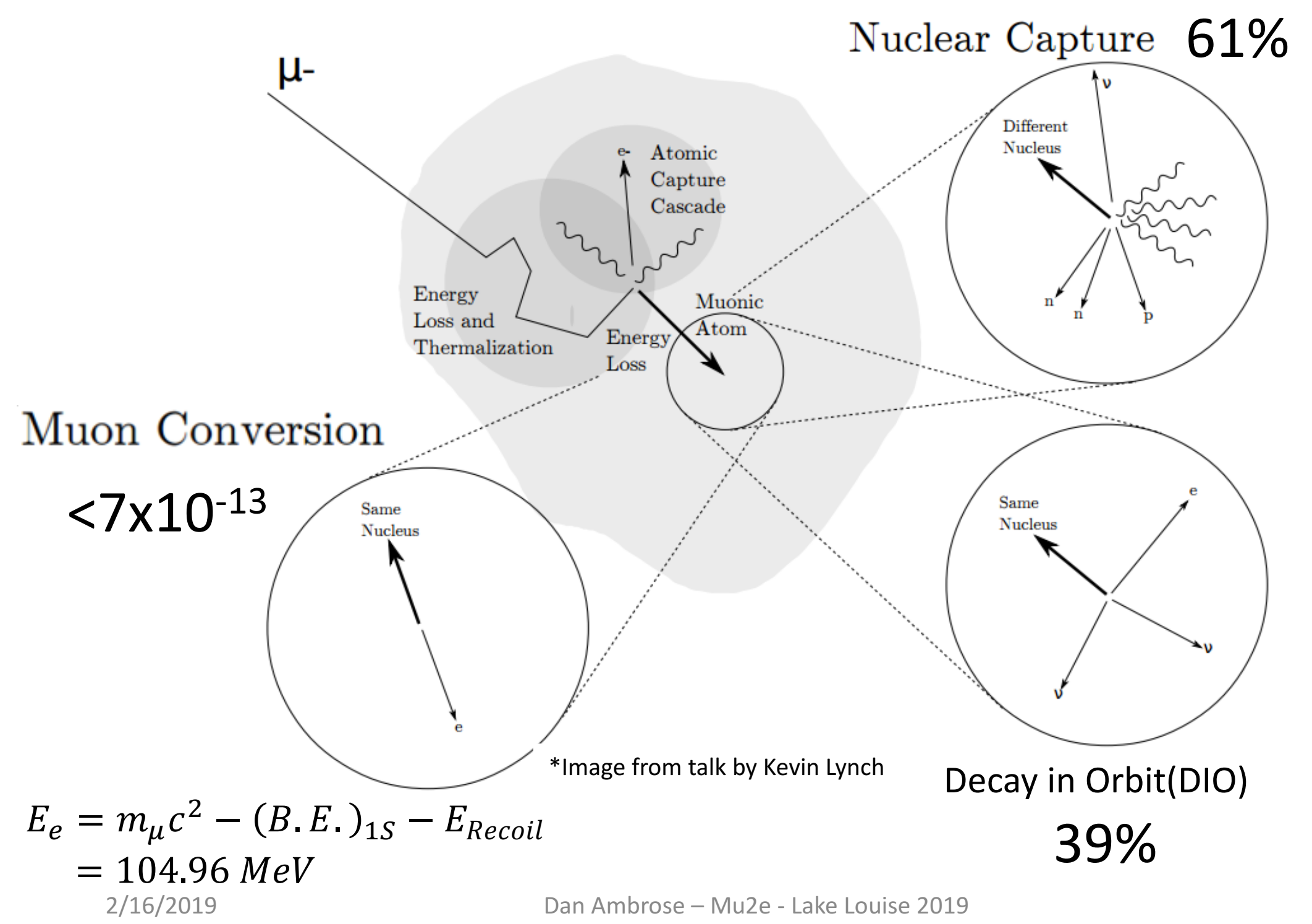




\section{Comparing DIO to conversion electrons}

Free muon decay

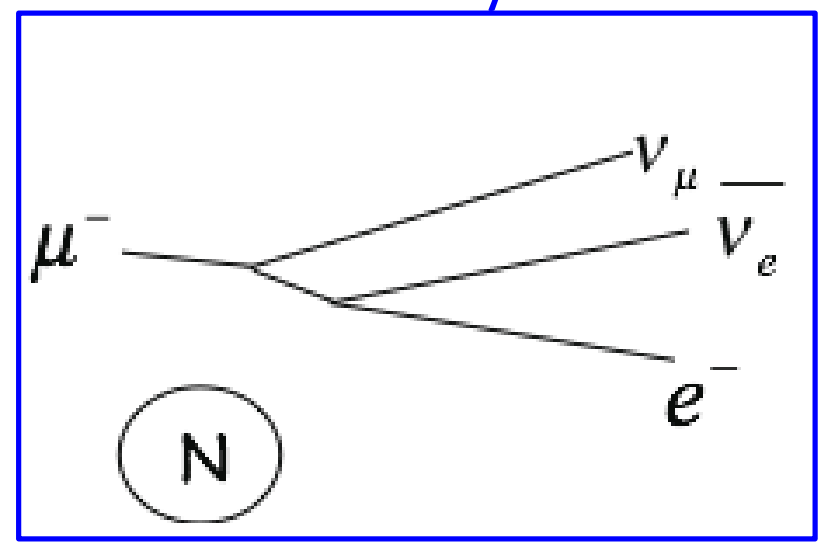

Nucleus affected DIO shape

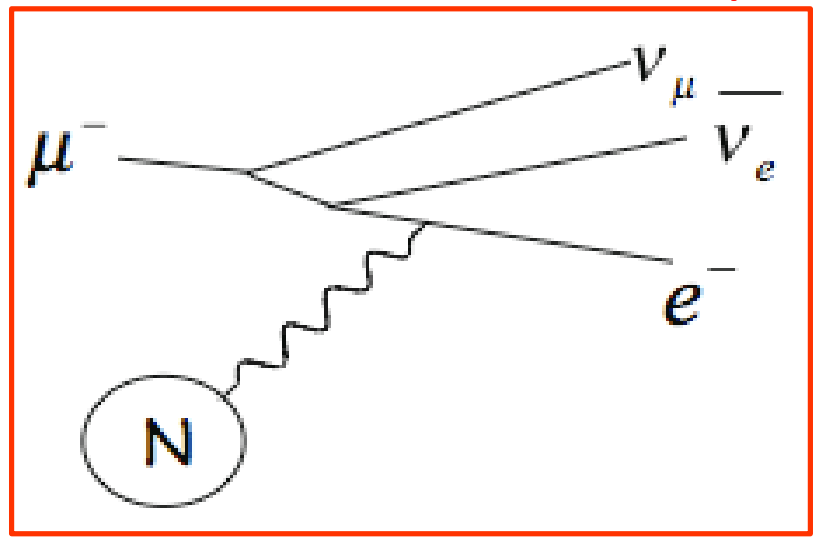

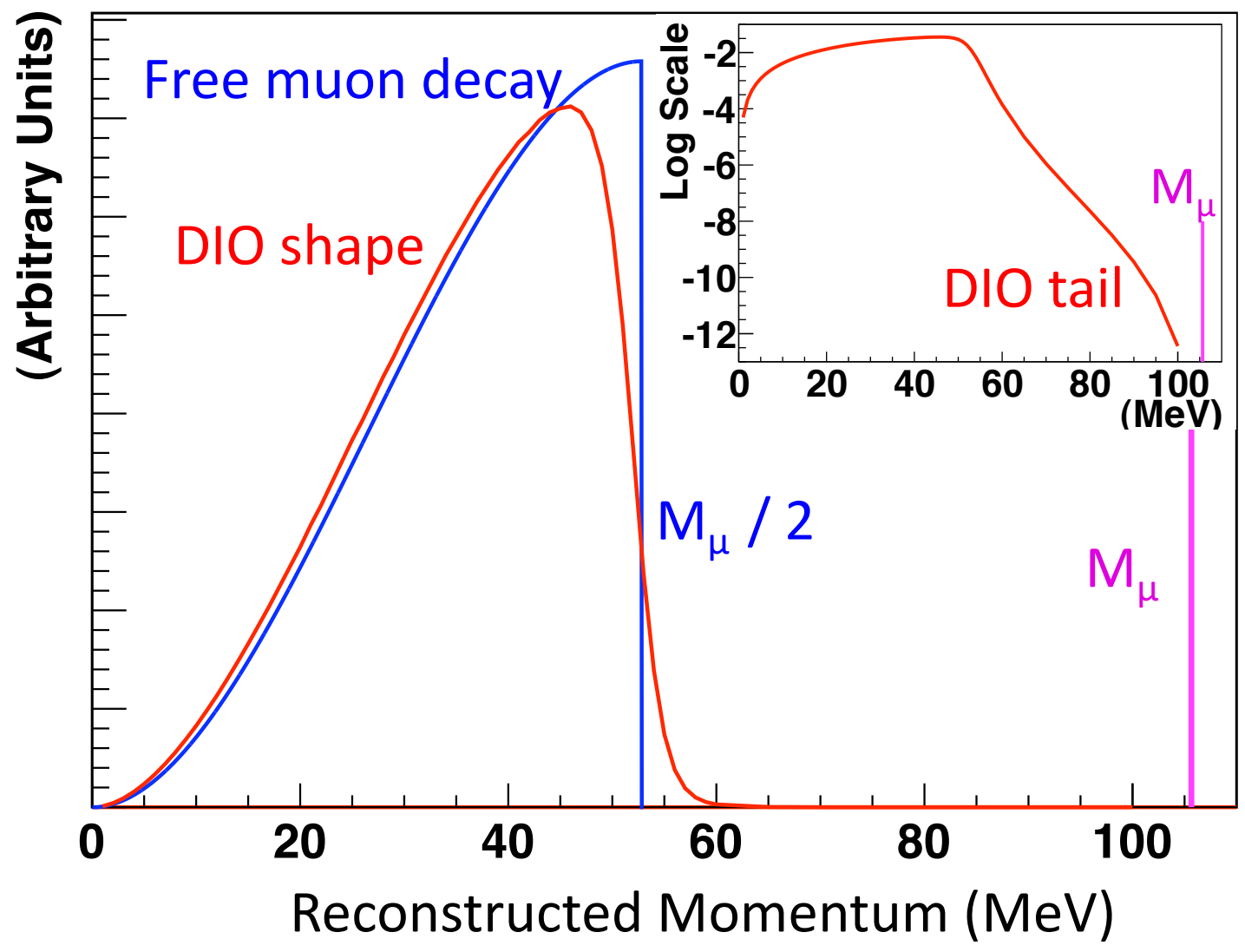


Experimental effects on

\section{DIO to conversion electrons}

- Tail of DIO falls as $\left(E_{\text {Endpoint }}-E_{e}\right)^{5}$

- A window of a few hundred keV, where Signal >> DIO for $R_{\mu e}=10^{-16}$
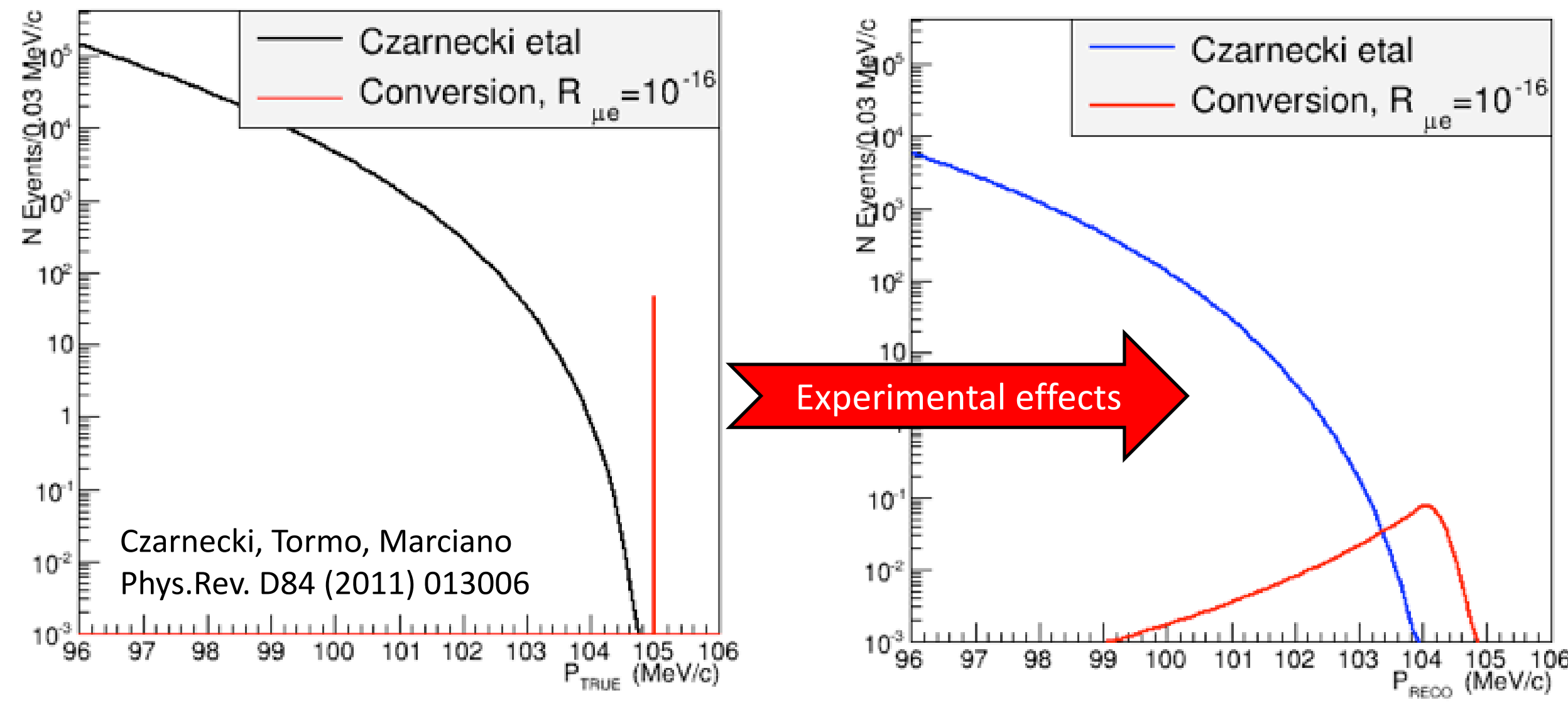


\section{Fermilab Facility: The Proton's Path}

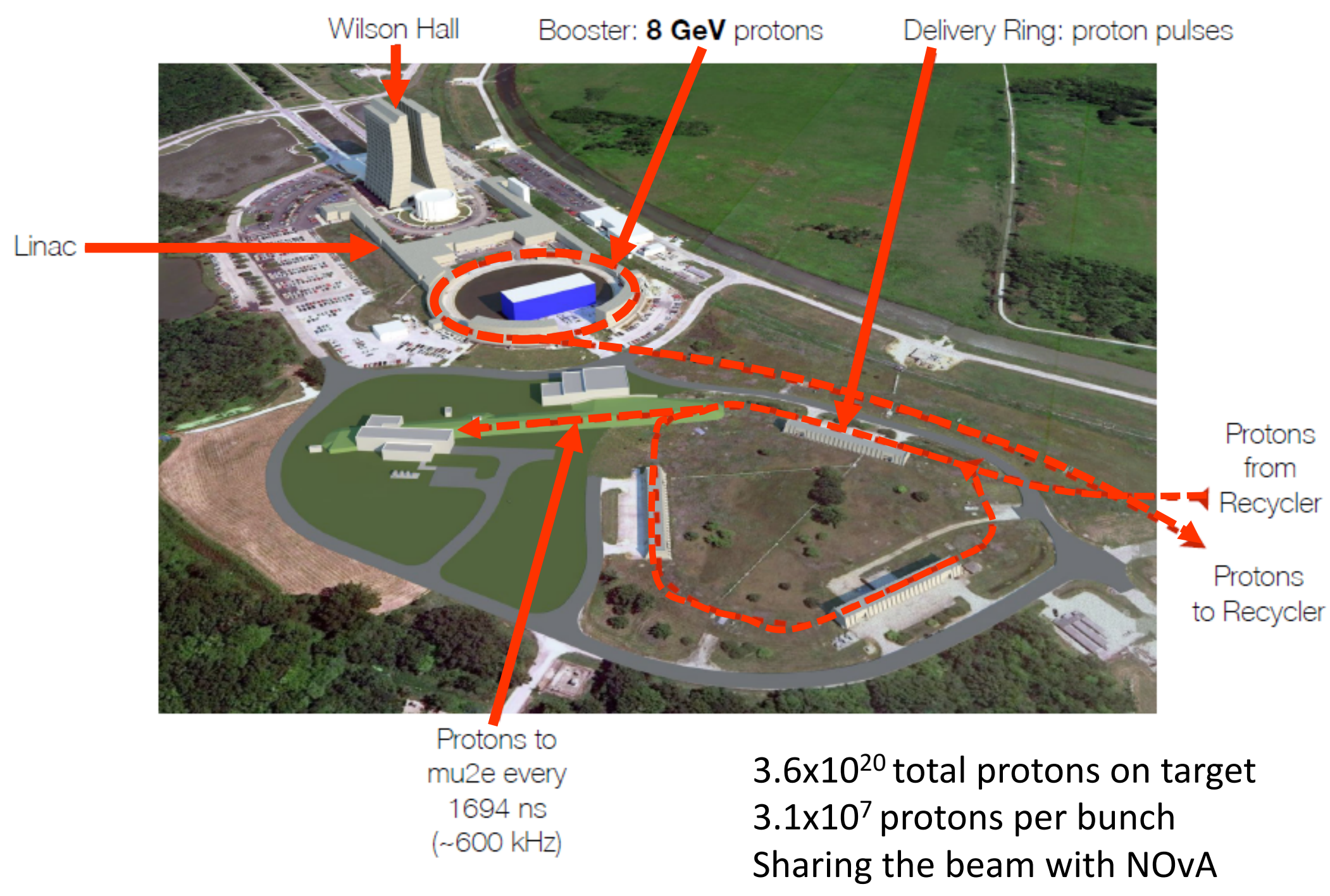




\section{Beam Time}

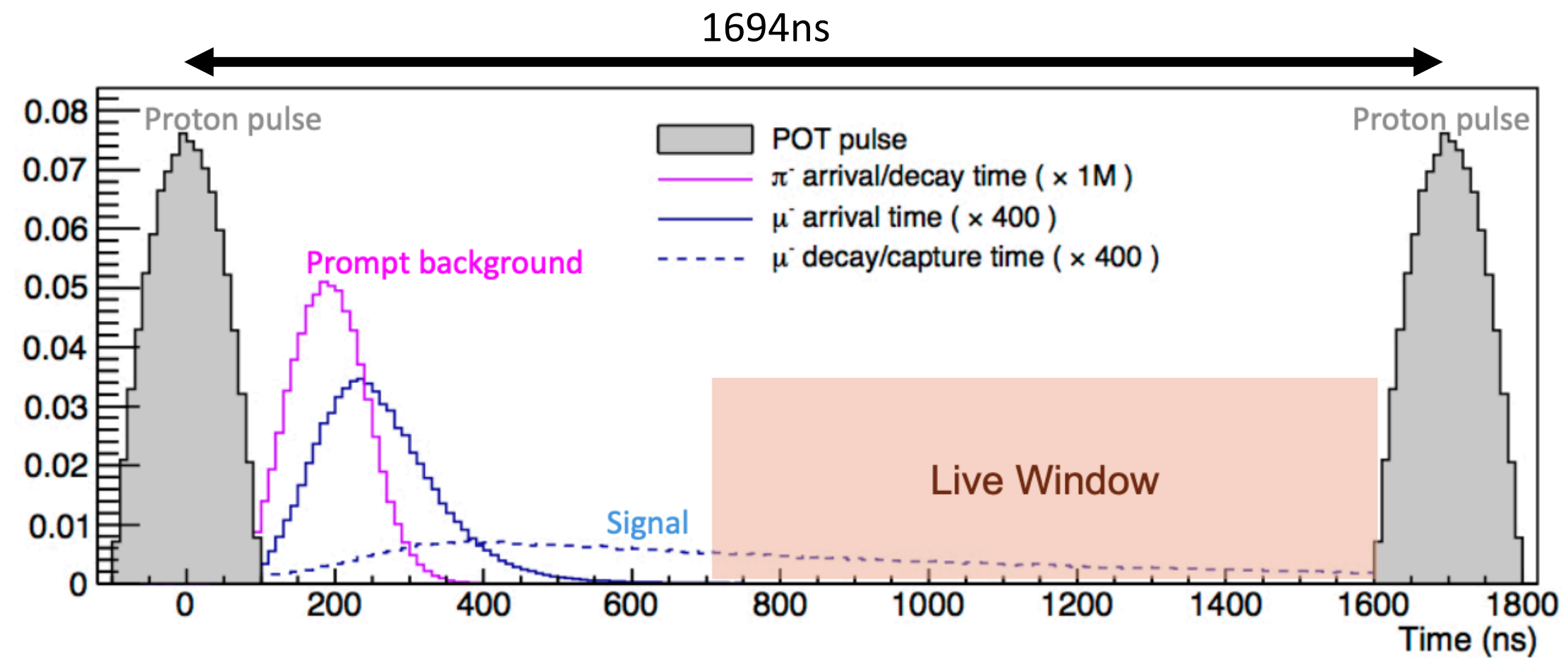

- 20,000 muons per bunch

- $10^{10}$ muons per second

Almost all protons, unstopped muons, stopped and unstopped pions will have passed through the detector before observation window. 


\section{Annular Detector Geometry}

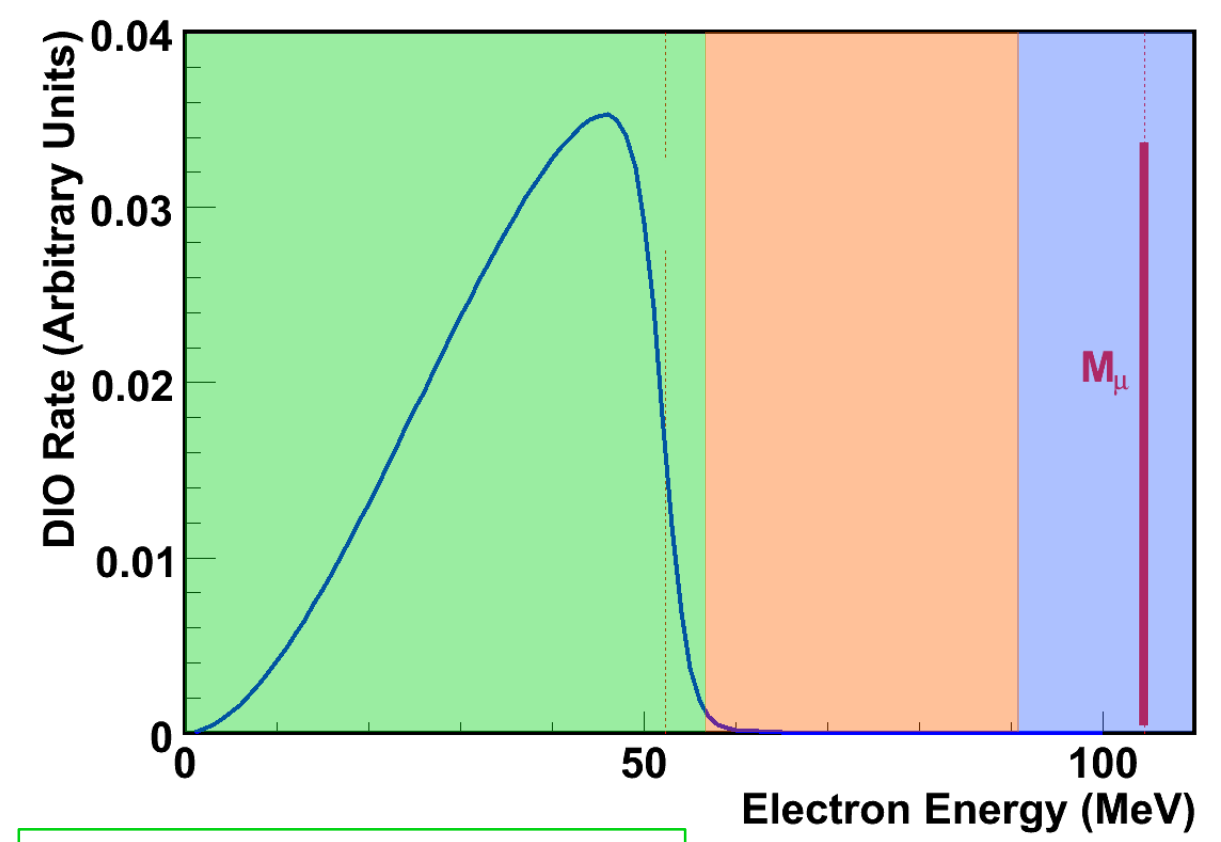

No hits in detector

Some hits in detector.

Tracks not reconstructable.

Reconstructable tracks Momentum resolution $\sigma<180 \mathrm{keV} / \mathrm{c}$ 


\section{Low Mass Tracker Design}

- Excellent momentum

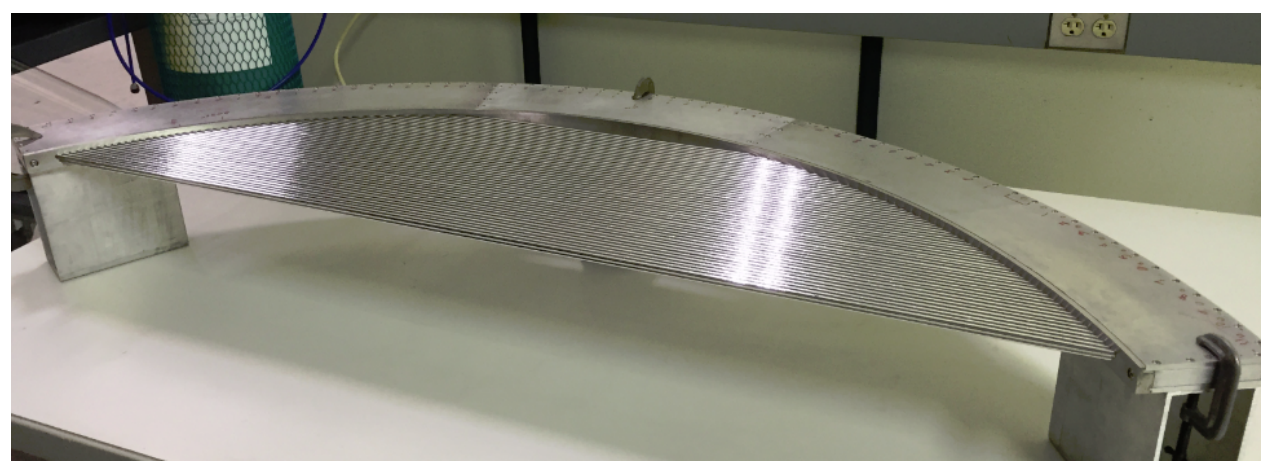
resolution better than 180 $\mathrm{KeV} / \mathrm{c}$

- 15 micron thick Mylar straws provide for a very low mass detector
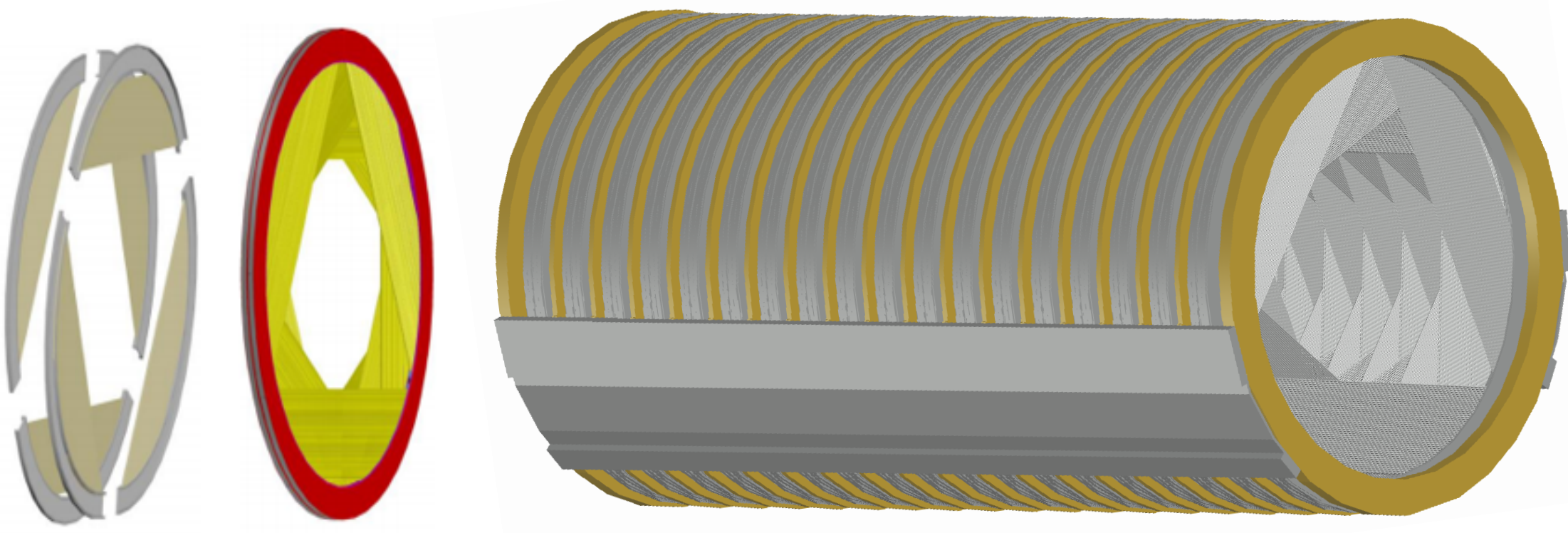


\section{Calorimeter}

- Provides precise timing, PID, seed for tracking and triggering

- Will employ 2 disks (radius $=37-66 \mathrm{~cm}$ )

- 1400 Csl crystals with square cross-section $\sim 3 \mathrm{~cm}$ diameter, $\sim 20$
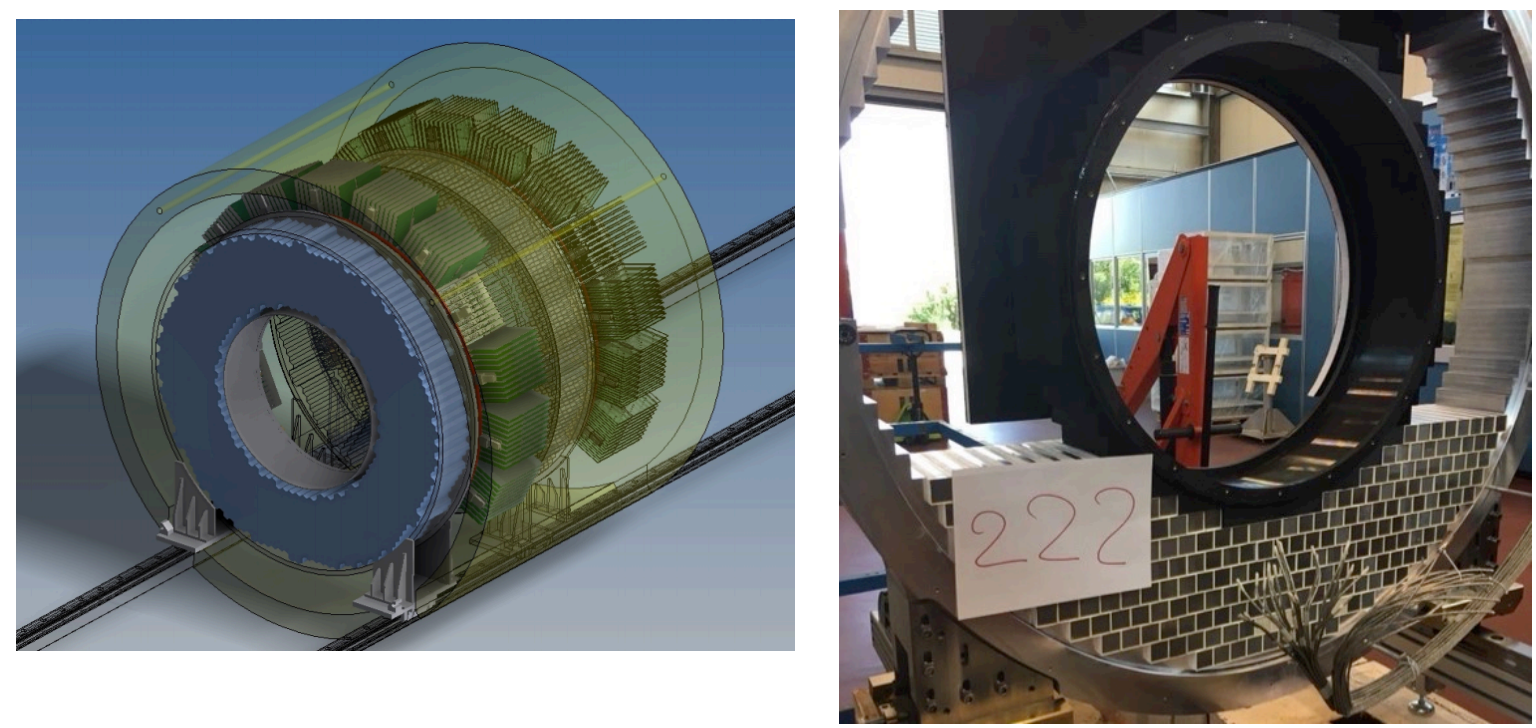
$\mathrm{cm}$ long (10 X0)

- Calorimeter properties have been measured in beam tests
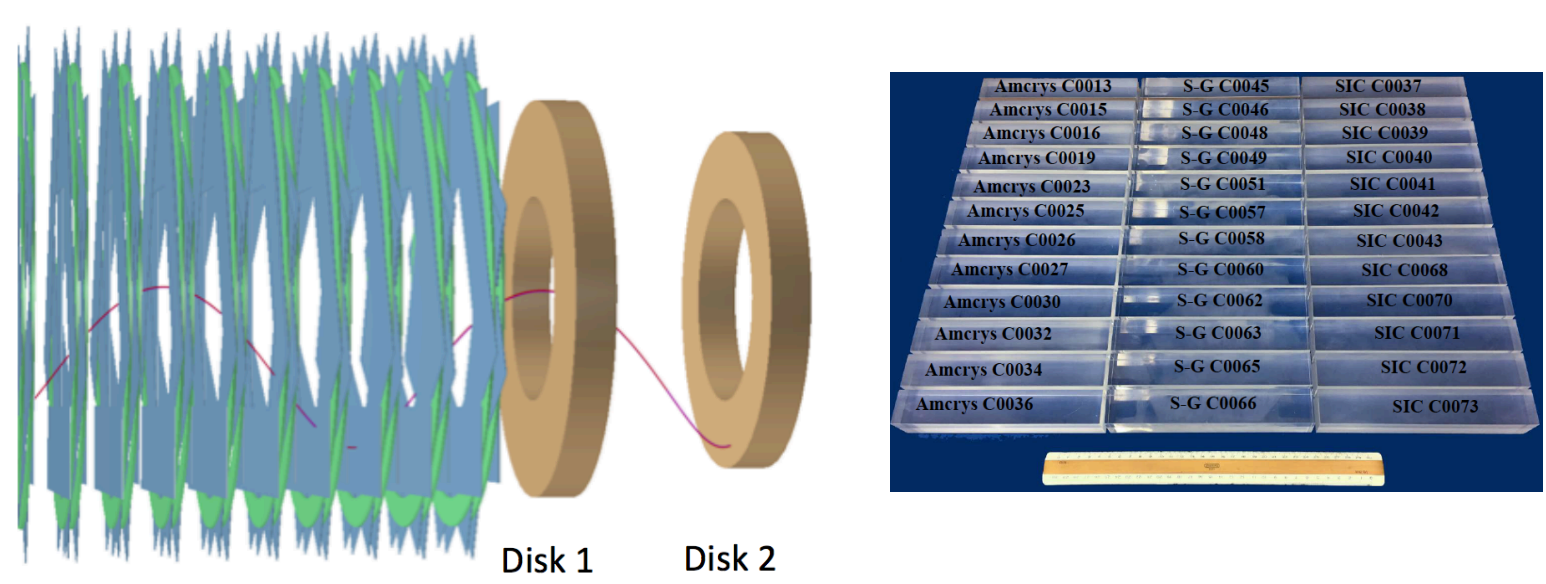


\section{Cosmic Ray Veto (CRV)}

- The CRV covers all of DS and half of TS

- Without CRV, expect 1 cosmic-ray-induced background event per day $(99.99 \%$ net efficiency)

- The CRV consists of 4 overlapping layers of scintillator strips with wavelength shifter and aluminum absorber

- Have achieved $\varepsilon>99.4 \%$ (per layer) in test beam
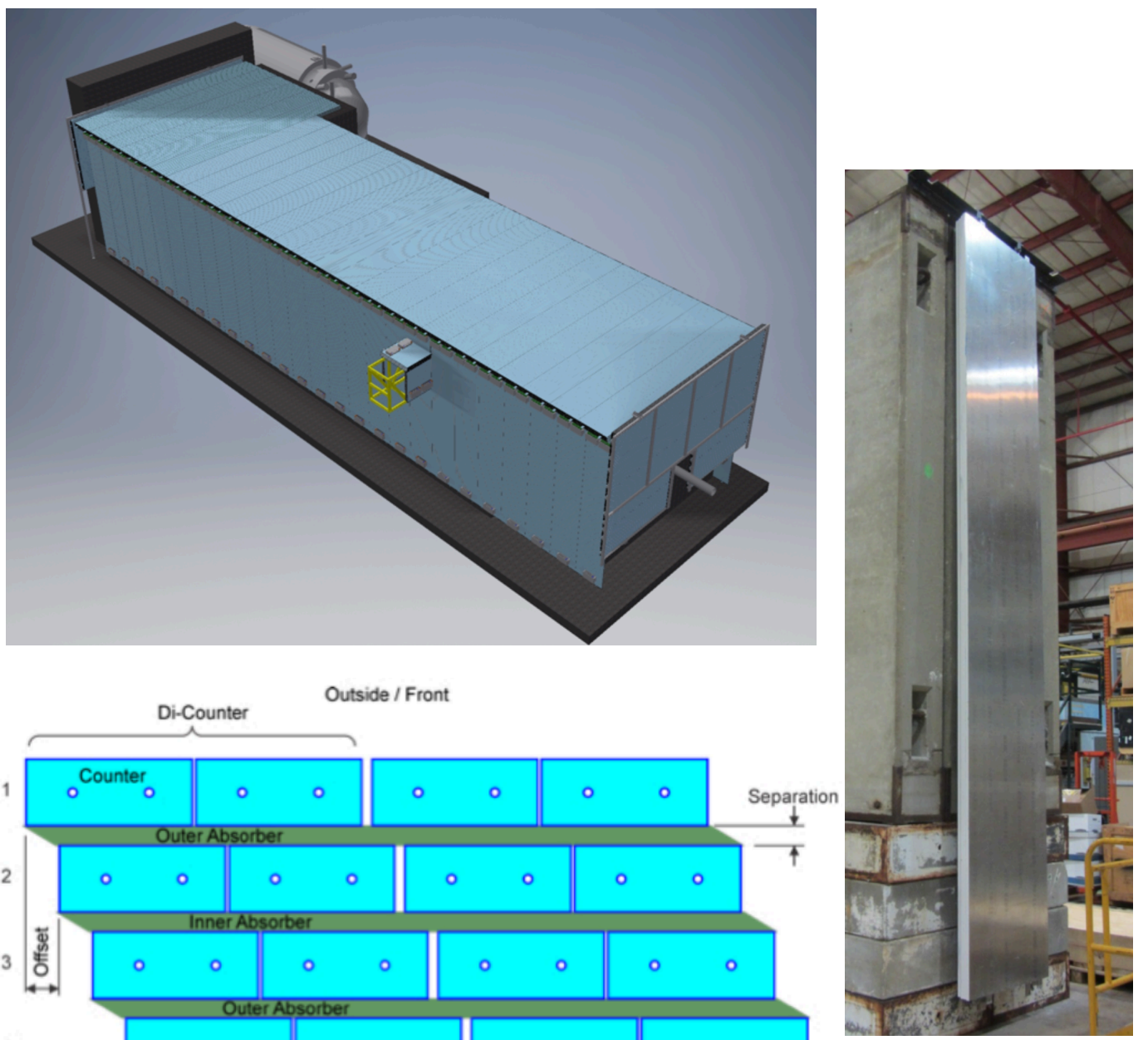


\section{Estimated background yields}

Category

Intrinsic *

Late Arriving $^{+}$

Miscellaneous

Late Atriving

Miscellaneous

Background process

Estimated yield

(events)

\section{Muon decay-in-orbit (DIO) \\ Muon capture (RMC)}

Pion capture (RPC)

Muon decay-in-flight ( $\mu$-DIF)

Pion decay-in-flight ( $\pi$-DIF)

Beam electrons

Antiproton induced

Cosmic ray induced

$$
\begin{aligned}
& 0.144 \pm 0.028 \text { (stat) } \pm 0.11 \text { (syst) } \\
& 0.000_{-0.000}^{+0.004}
\end{aligned}
$$$$
0.021 \pm 0.001 \text { (stat) } \pm 0.002 \text { (syst) }
$$$$
<0.003
$$

$0.001 \pm<0.001$

$(2.1 \pm 1.0) \times 10^{-4}$

$$
\begin{aligned}
& 0.040 \pm 0.001 \text { (stat) } \pm 0.020 \text { (syst) } \\
& 0.209 \pm 0.022 \text { (stat) } \pm 0.055 \text { (syst) }
\end{aligned}
$$

Total $\quad 0.41 \pm 0.13$ (stat+syst)

*Assuming $6 \times 10^{17}$ stopped muons in $6 \times 10^{7}$ sec of beam time +Assuming an proton beam extinction of $10^{-10}$, a cosmic ray veto inefficiency of $10^{-4}$, PID muon-rejection of 200. 


\section{Reconstruction Simulation}

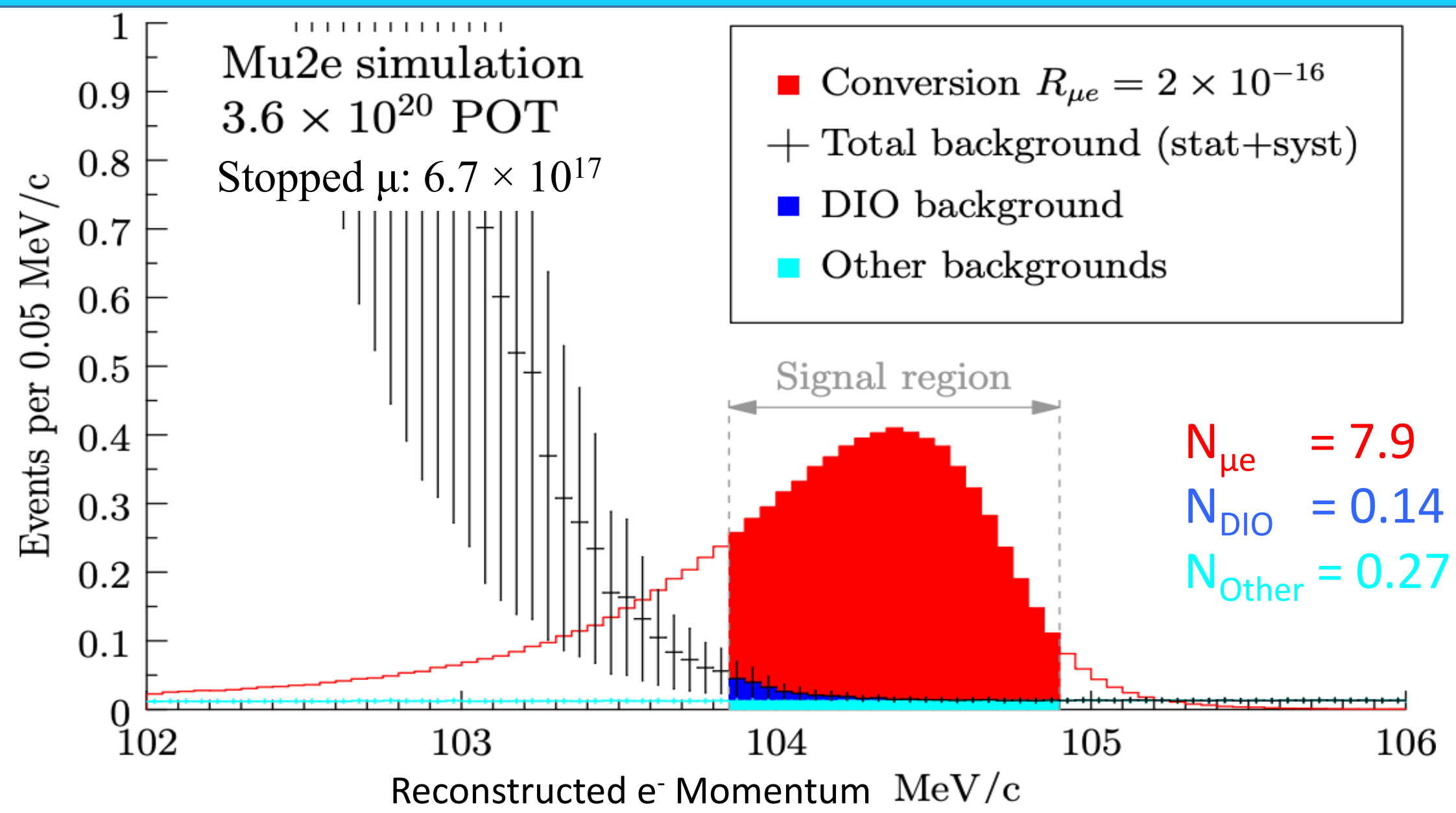

This simulation includes measured properties of prototype detectors.
At $R_{\mu e}=2 \times 10^{-16}$, we would expect to see 7.9 events which is a $5 \sigma$ discovery! 


\section{Production is underway!}
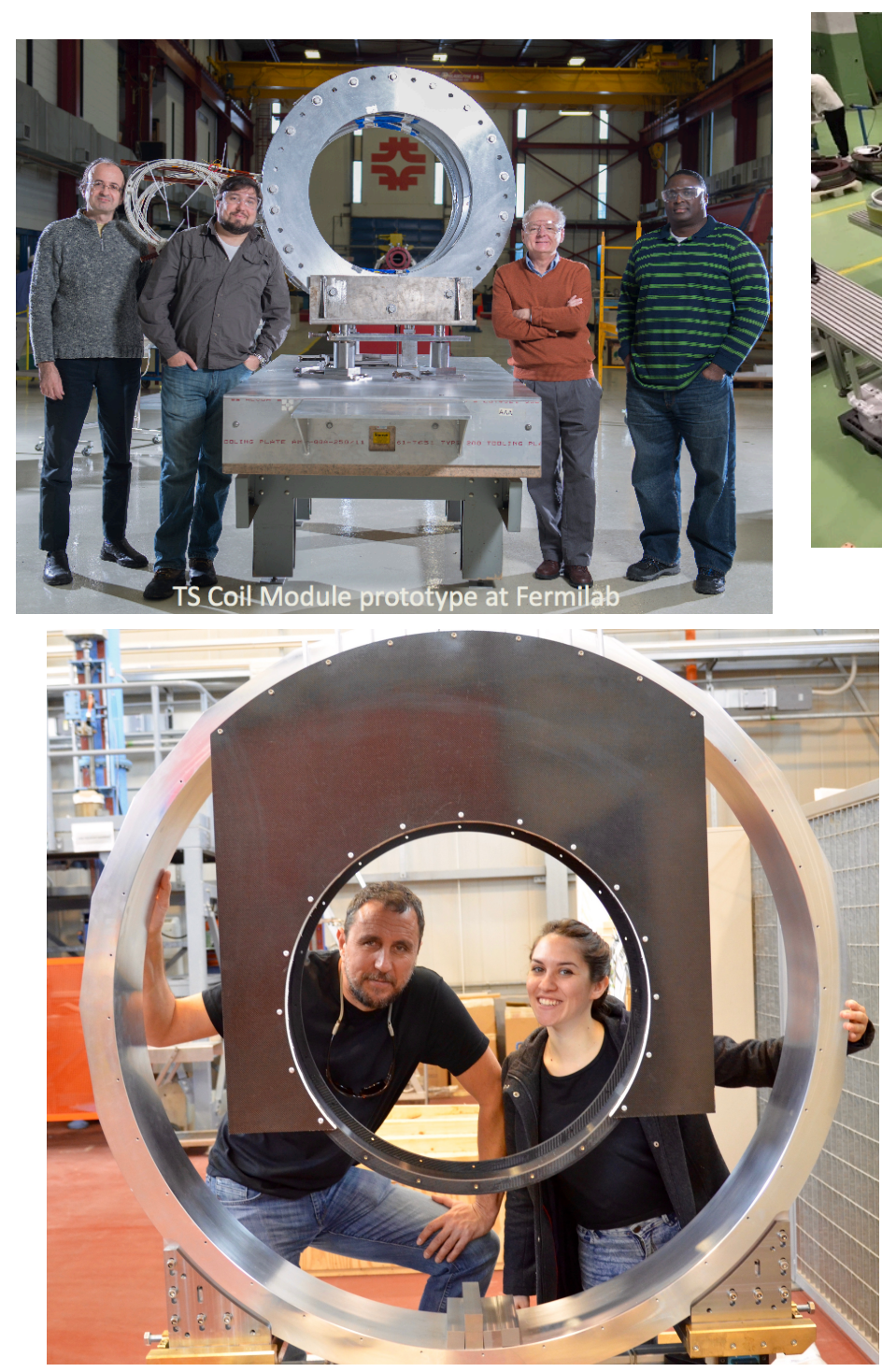

2/16/2019
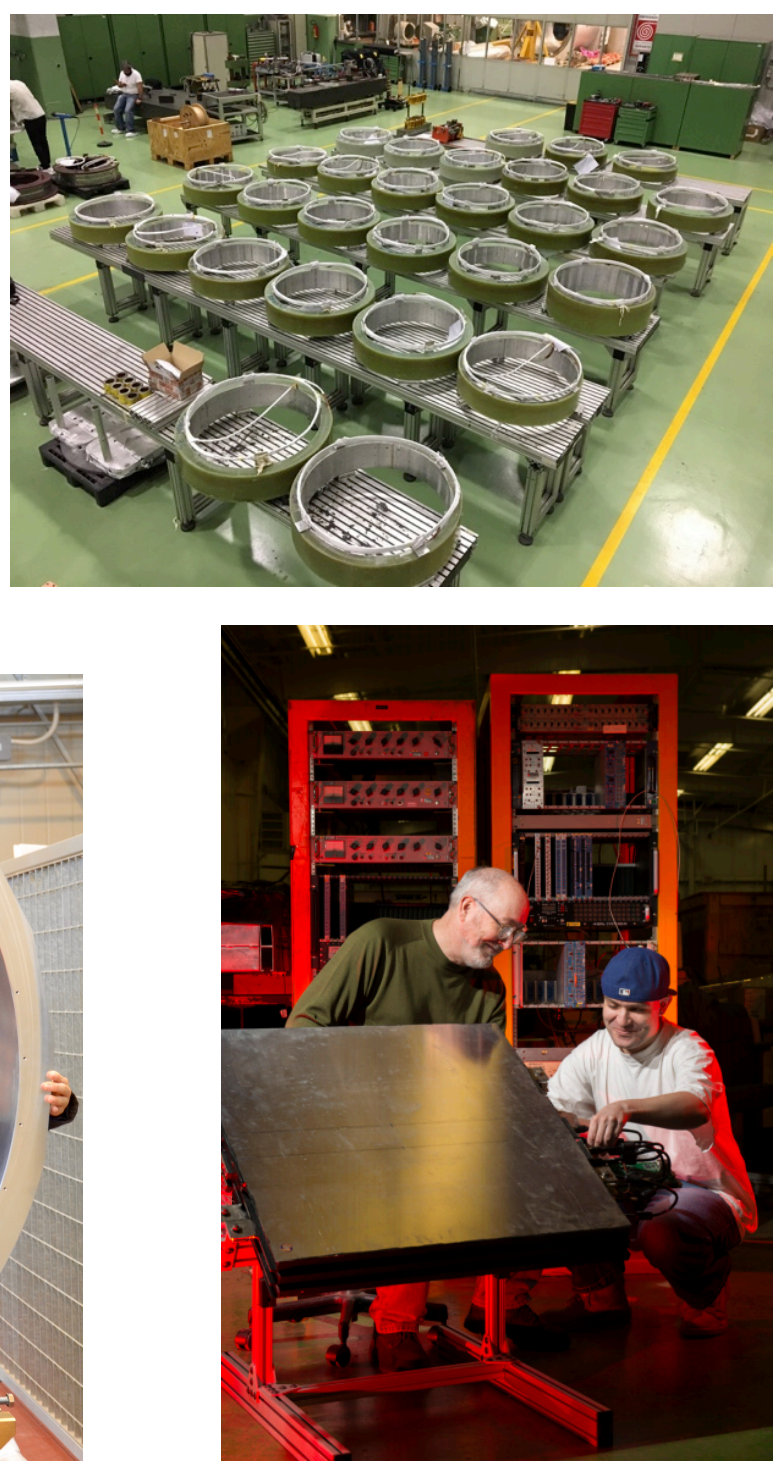

Dan Ambrose - Mu2e - Lake Louise 2019
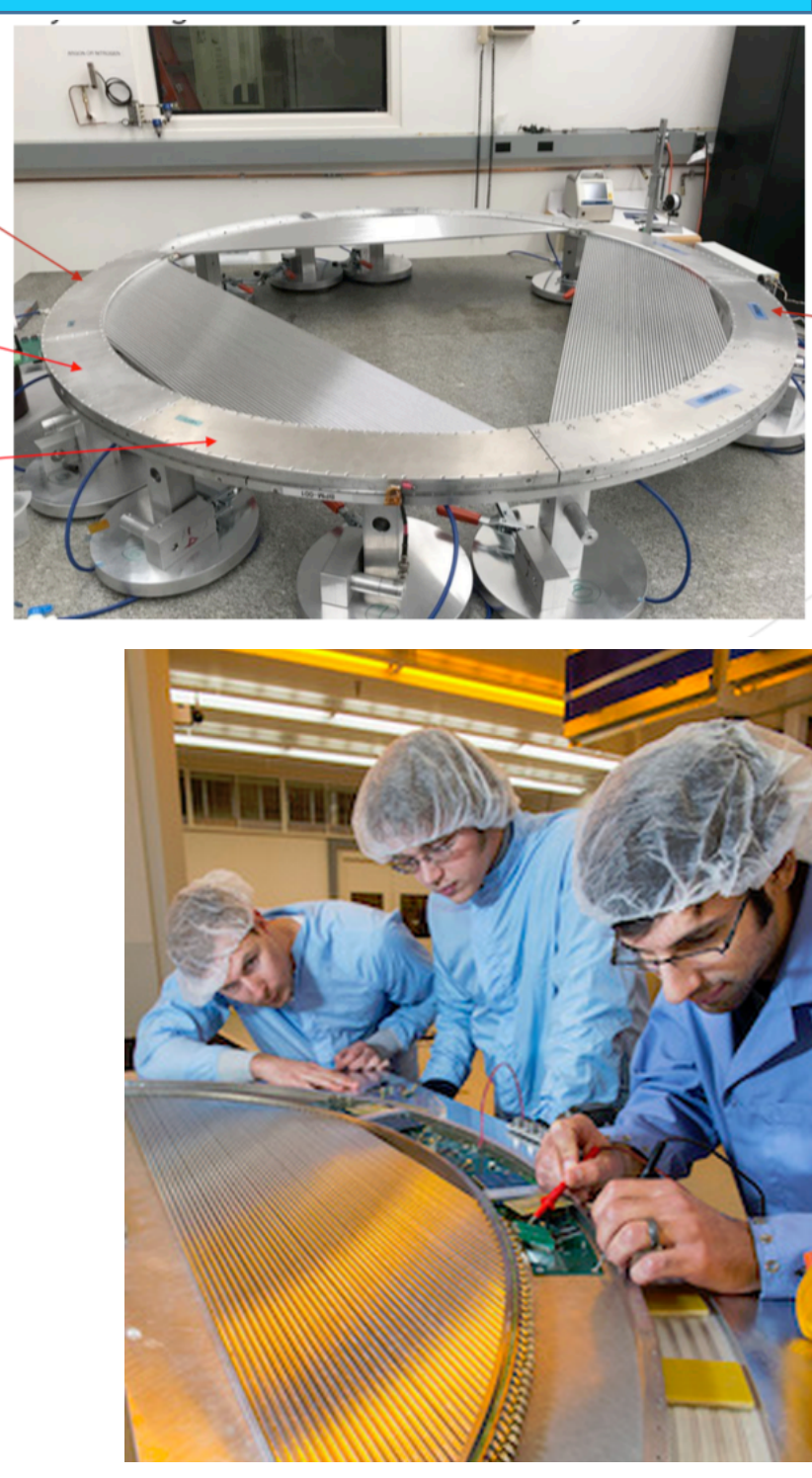


\section{New Building completed in 2016}
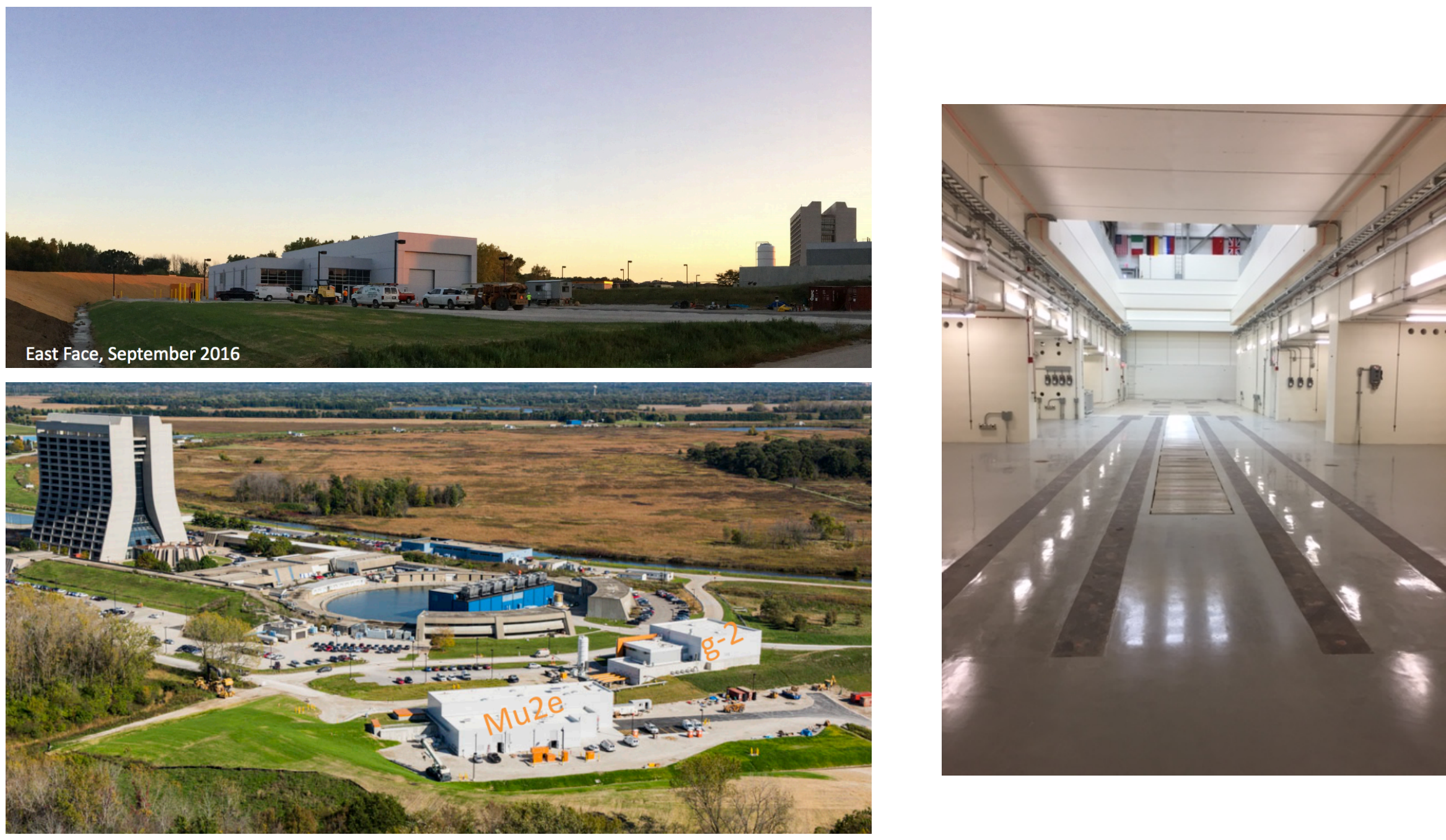


\section{Schedule}

Mu2e is on schedule.

We expect to be commissioning in 2020 .

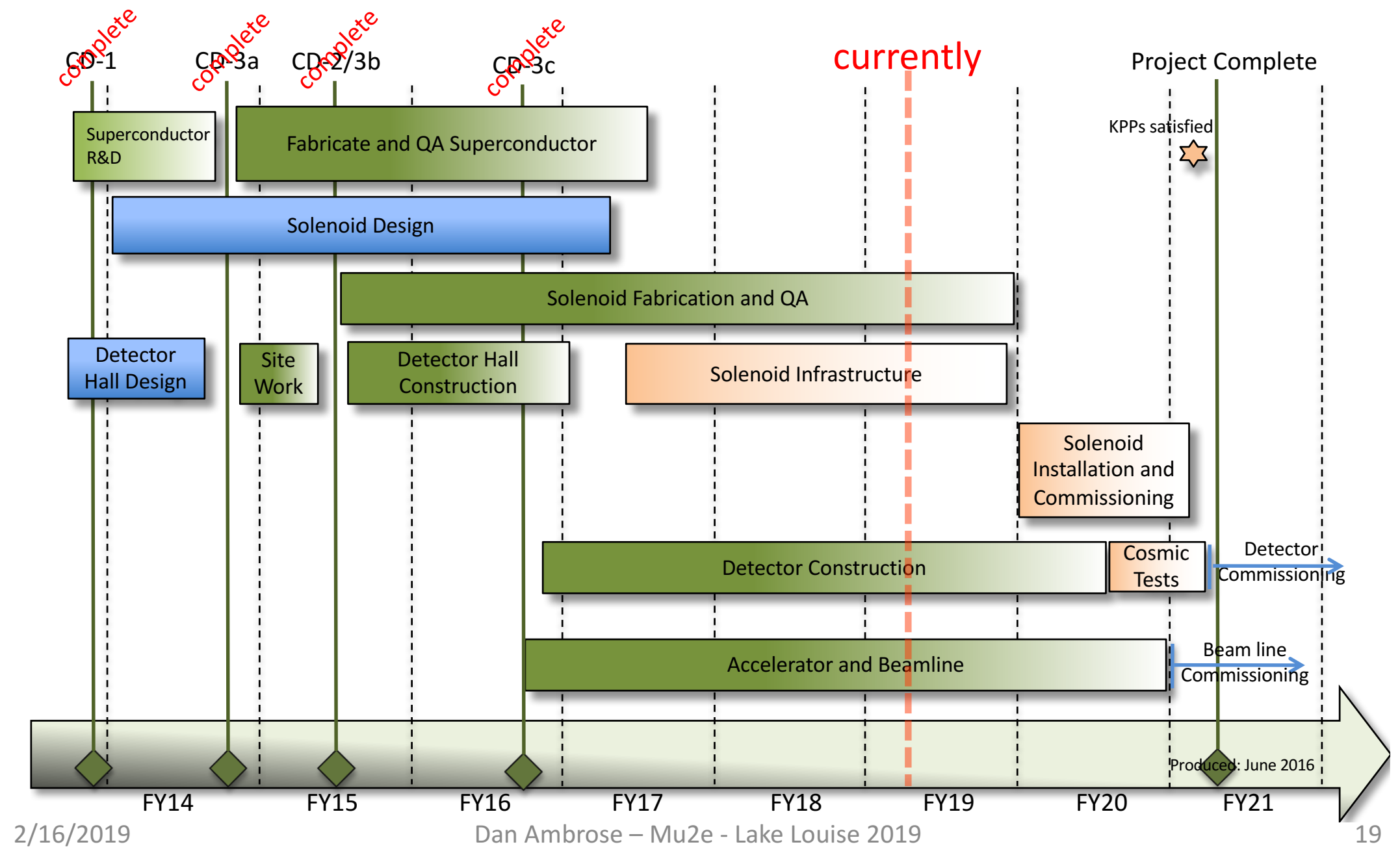




\section{The Muze Collaboration}

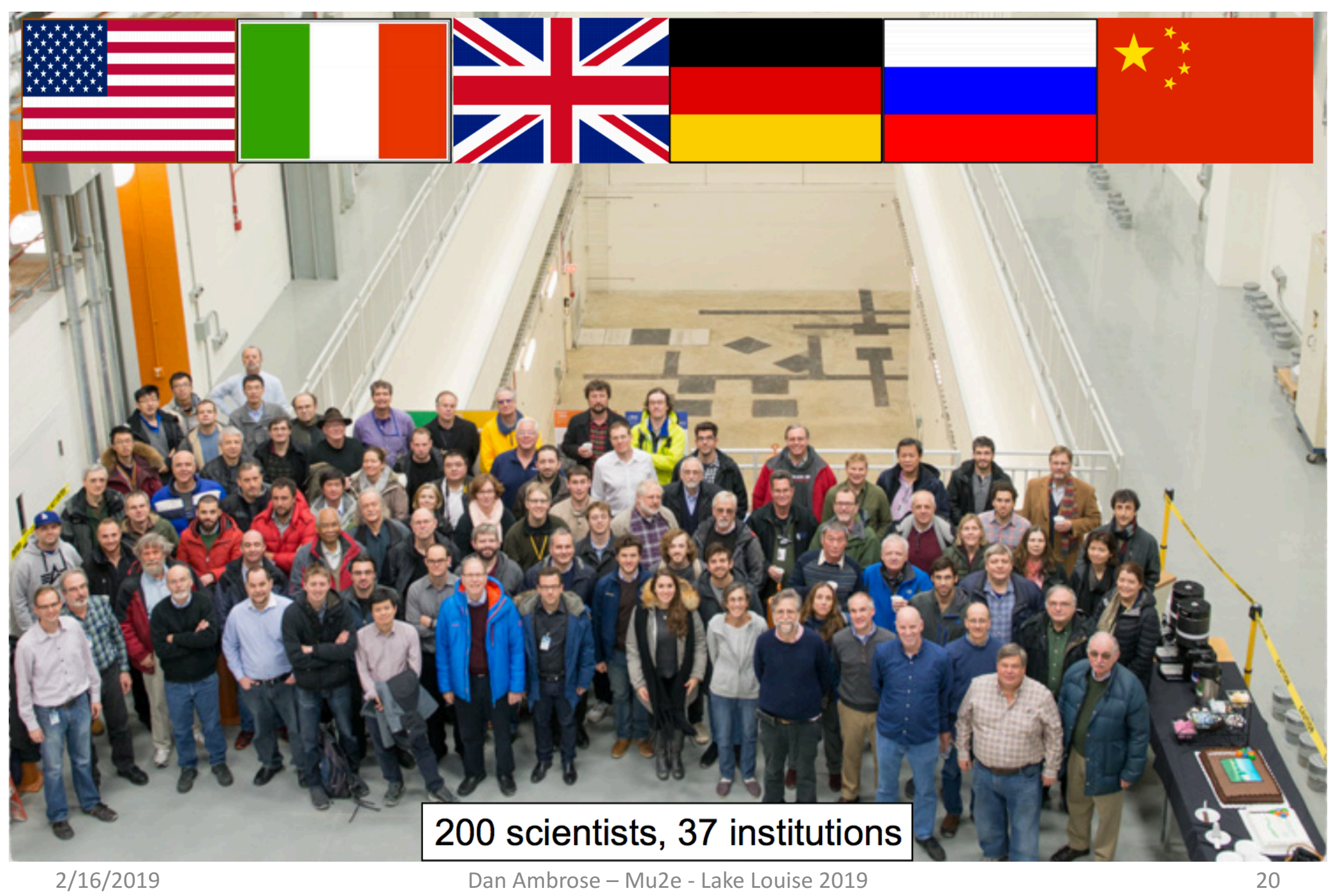




\section{Summary}

- There is a lot of excitement about theoretical model discrimination afforded by a 4 order of magnitude improvement on muon conversion sensitivity.

- The Mu2e project has a design which will allow for a single event sensitivity of $2.5 \times 10^{-17}$.

- The experimental design is mature and on schedule for commissioning in 2020 and taking production data starting in 2021.

For more detailed information ask or check out :

Summary of Experiment http://arxiv.org/pdf/1901.11099.pdf

Technical Design Report http://arXiv.org/abs/1501.05241 Experiment web site http://mu2e.fnal.gov

- A plan for the upgrade, Mu2e-II, has also progressed

Expression of Interest http://arxiv.org/pdf/1802.02599.pdf 


\section{Thank you}




\section{Sensitivity to High Mass Scales}

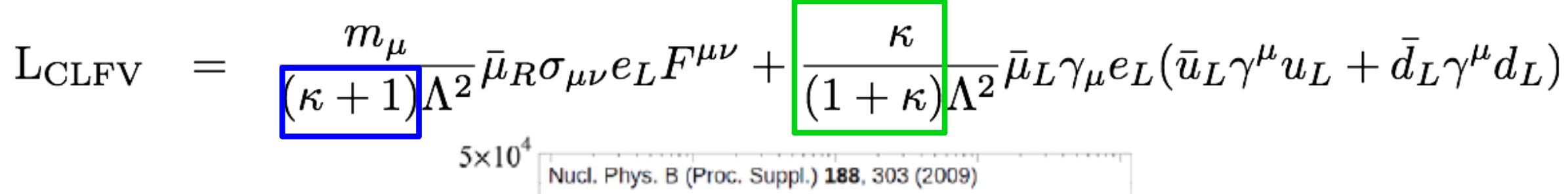

Loops dominate for $\mathrm{k}<1$

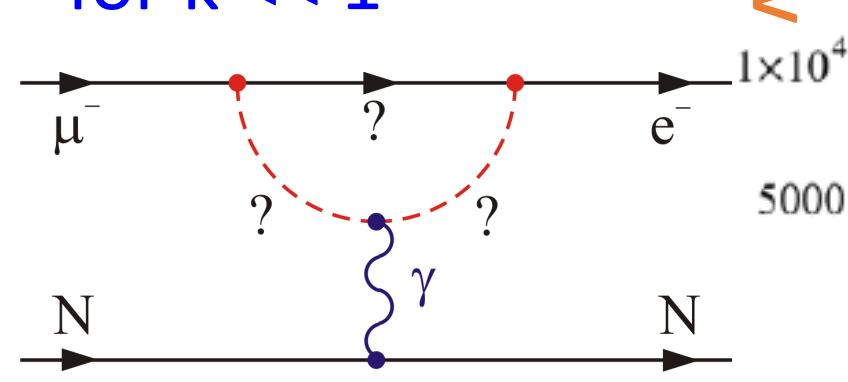

$\mu \rightarrow \mathrm{e} \psi$

$\mu \mathrm{N} \rightarrow \mathrm{eN}$

$\mu \rightarrow$ eee all limits @ 90\% CL

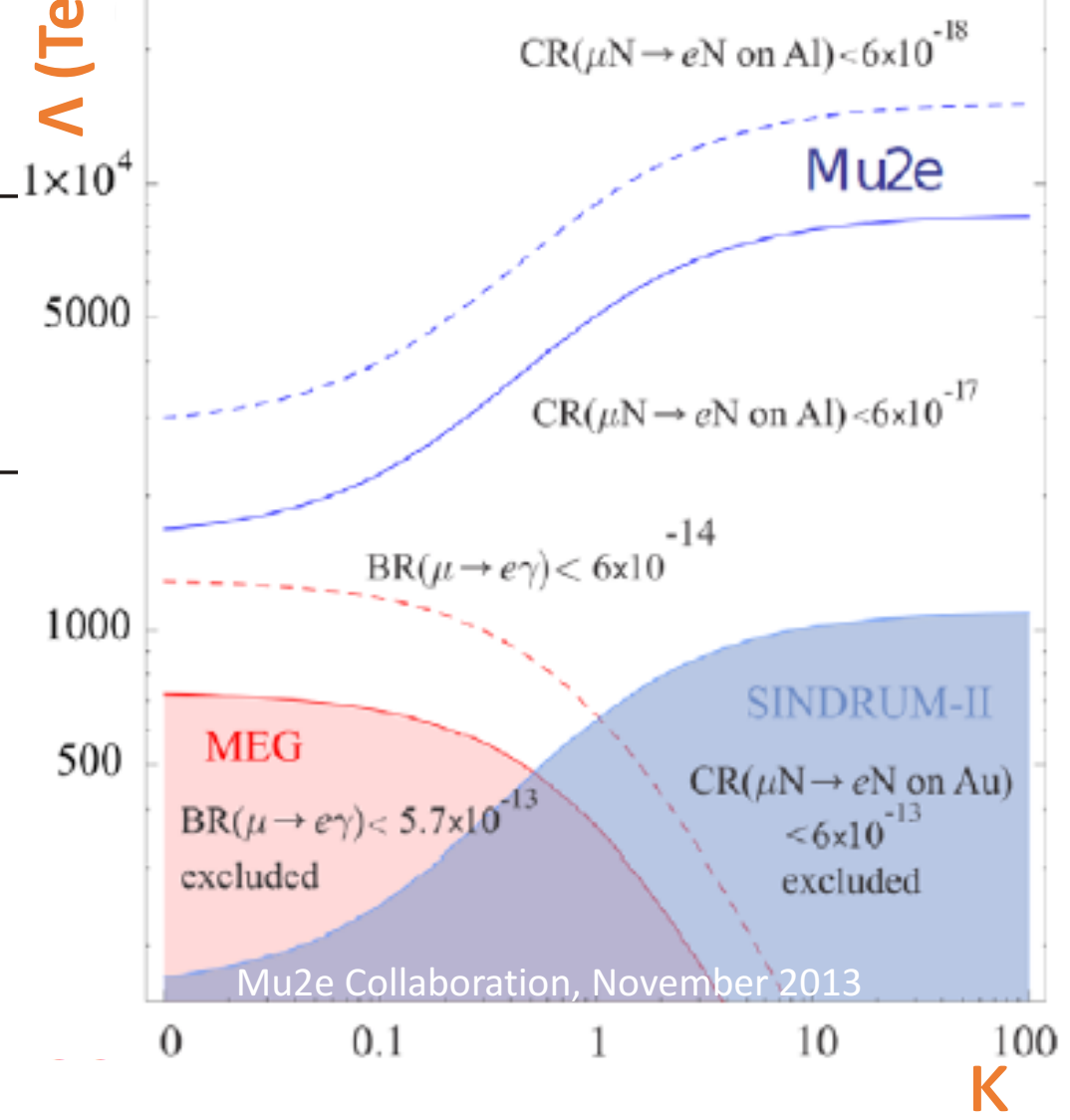

Dan Ambrose - Mu2e - Lake Louise 2019
Contact terms dominate for $\mathrm{k}>>1$

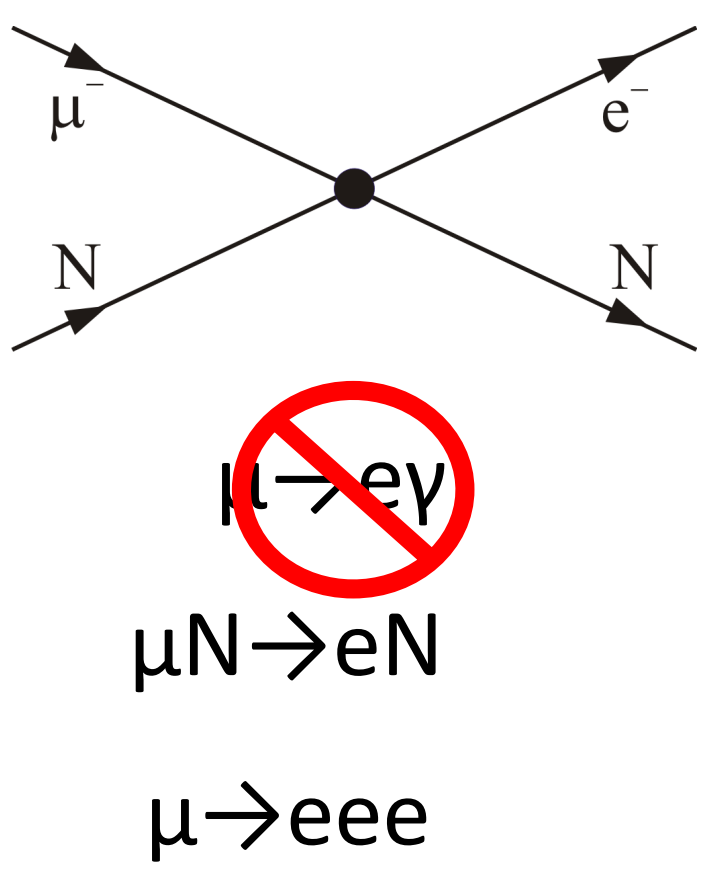




\section{Sensitive to many models}

\section{$\Lambda_{c}$ between 2000 to $7000 \mathrm{TeV}$}

\section{Supersymmetry}

rate $\sim 10^{-15}$

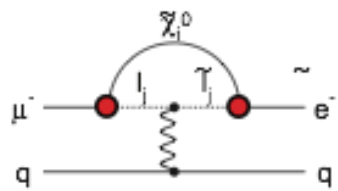

Heavy Neutrinos

$\left|\mathrm{U}_{\mu N} \mathrm{U}_{\mathrm{eN}}\right|^{2} \sim 8 \times 10^{-13}$

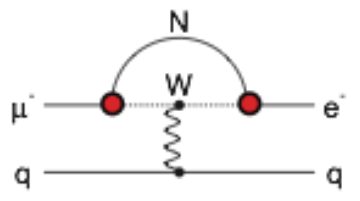

\section{Compositeness}

$\Lambda_{\mathrm{c}} \sim 3000 \mathrm{TeV}$

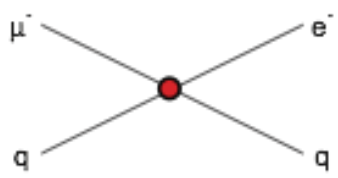

Leptoquark

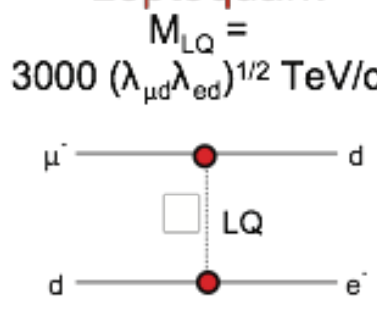

Heavy Z'

Second Higgs Doublet

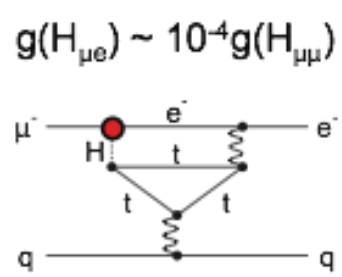

W. Altmannshofer, A.J.Buras, S.Gori, P.Paradisi, D.M.Straub

\begin{tabular}{|l|c|c|c|c|c|c|c|}
\hline & AC & RVV2 & AKM & $\delta$ LL & FBMSSM & LHT & RS \\
\hline \hline$D^{0}-\bar{D}^{0}$ & $\star \star \star$ & $\star$ & $\star$ & $\star$ & $\star$ & $\star \star \star$ & $?$ \\
\hline$\epsilon_{K}$ & $\star$ & $\star \star \star$ & $\star \star \star$ & $\star$ & $\star$ & $\star \star$ & $\star \star \star$ \\
\hline$S_{\psi \phi}$ & $\star \star \star$ & $\star \star \star$ & $\star \star \star$ & $\star$ & $\star$ & $\star \star \star$ & $\star \star \star$ \\
\hline \hline$S_{\phi K_{S}}$ & $\star \star \star$ & $\star \star$ & $\star$ & $\star \star \star$ & $\star \star \star$ & $\star$ & $?$ \\
\hline$A_{\mathrm{CP}}\left(B \rightarrow X_{s} \gamma\right)$ & $\star$ & $\star$ & $\star$ & $\star \star \star$ & $\star \star \star$ & $\star$ & $?$ \\
\hline$A_{7,8}\left(B \rightarrow K^{*} \mu^{+} \mu^{-}\right)$ & $\star$ & $\star$ & $\star$ & $\star \star \star$ & $\star \star \star$ & $\star \star$ & $?$ \\
\hline$A_{9}\left(B \rightarrow K^{*} \mu^{+} \mu^{-}\right)$ & $\star$ & $\star$ & $\star$ & $\star$ & $\star$ & $\star$ & $?$ \\
\hline$B \rightarrow K^{(*)} \nu \bar{\nu}$ & $\star$ & $\star$ & $\star$ & $\star$ & $\star$ & $\star$ & $\star$ \\
\hline$B_{s} \rightarrow \mu^{+} \mu^{-}$ & $\star \star \star$ & $\star \star \star$ & $\star \star \star$ & $\star \star \star$ & $\star \star \star$ & $\star$ & $\star$ \\
\hline$K^{+} \rightarrow \pi^{+} \nu \bar{\nu}$ & $\star$ & $\star$ & $\star$ & $\star$ & $\star$ & $\star \star \star$ & $\star \star \star$ \\
\hline$K_{L} \rightarrow \pi^{0} \nu \bar{\nu}$ & $\star$ & $\star$ & $\star$ & $\star$ & $\star$ & $\star \star \star$ & $\star \star \star$ \\
\hline$\mu \rightarrow e \gamma$ & $\star \star \star$ & $\star \star \star$ & $\star \star \star$ & $\star \star \star$ & $\star \star \star$ & $\star \star \star$ & $\star \star \star$ \\
\hline$\tau \rightarrow \mu \gamma$ & $\star \star \star$ & $\star \star \star$ & $\star$ & $\star \star \star$ & $\star \star \star$ & $\star \star \star$ & $\star \star \star$ \\
\hline$\mu+N \rightarrow e+N$ & $\star \star \star$ & $\star \star \star$ & $\star \star \star$ & $\star \star \star$ & $\star \star \star$ & $\star \star \star$ & $\star \star \star$ \\
\hline \hline$d_{n}$ & $\star \star \star$ & $\star \star \star$ & $\star \star \star$ & $\star \star$ & $\star \star \star$ & $\star$ & $\star \star \star$ \\
\hline$d_{e}$ & $\star \star \star$ & $\star \star \star$ & $\star \star$ & $\star$ & $\star \star \star$ & $\star$ & $\star \star \star$ \\
\hline$(g-2)_{\mu}$ & $\star \star \star$ & $\star \star \star$ & $\star \star$ & $\star \star \star$ & $\star \star \star$ & $\star$ & $?$ \\
\hline
\end{tabular}

Table 8: "DNA" of flavour physics effects for the most interesting observables in a selection of SUSY and non-SUSY models $\star \star \star$ signals large effects, $\star \star$ visible but small effects and $\star$ implies that

the given model does not predict sizable effects in that observable. 


\section{The Comet Experiment}

The Comet experiment is another muon to electron conversion experiment which will run at J-PARC.

Phase 1 will be a 100x sensitivity improvement is scheduled to begin in 2020 . Phase 2 has a similar sensitivity to Mu2e $\left(10^{4} x\right.$ sensitivity improvement) and is scheduled to begin after Mu2e begins.

For more information on the technical design of Comet : http://arxiv.org/pdf/1812.09018.pdf 